\title{
An Empirical Approach to the Prediction of the Channel Capacity in Ultra Wideband Indoor Propagation Channels
}

\author{
Christian Sturm* ${ }^{(1)}$, Ullrich J. Mönich ${ }^{(2)}$, \\ Thomas Zwick ${ }^{(1)}$, Werner Wiesbeck ${ }^{(1)}$, and Holger Boche ${ }^{(2)}$ \\ (1) Universität Karlsruhe (TH), Karlsruhe, Germany \\ (2) Technische Universität Berlin, Berlin, Germany \\ E-mail: christian.sturm@ihe.uka.de
}

\section{Introduction}

For radio network planning empirical channel models like the log distance path loss model are frequently applied. These models neglect the fading effects occurring due to multi-path propagation and hence are not applicable for predictions in rich scattering environments. Nevertheless, it has already been reported that for ultra wideband signals when assuming two-path propagation the received power does not show the classic fading characteristics but follows a log distance law [1]. In this paper the fading characteristics of ultra-wideband signals will be investigated for a real multi-path environment. It will be shown that also in this case the log distance law applies and a similar description can even be developed to describe the decrease of the channel capacity. A relation between the path loss exponent and the slope of this capacity decrease will be derived.

\section{Investigated Propagation Scenario}

With a ray-tracing based simulator the propagation of ultra wideband signals in a typical office room with outer dimensions of $7.27 \times 6.23 \times 3 \mathrm{~m}^{3}$ has been investigated. The applied ray tracer [2] resolves reflection paths up to 5th order and diffraction paths up to 2 nd order with full polarization information. For all objects in the scenario realistic dielectric and magnetic properties are assumed.

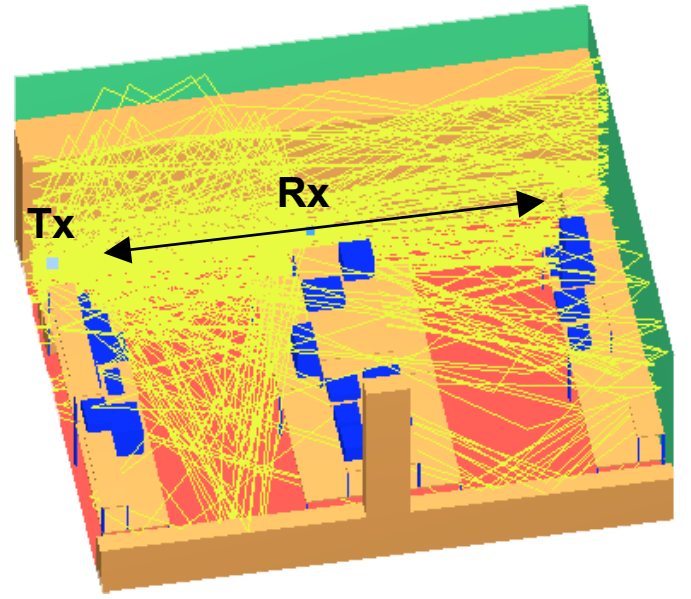

Figure 1: Investigated propagation scenario 
The investigated frequency range has been chosen according to the FCC ultra wideband regulations from 3.1 to $10.6 \mathrm{GHz}$. Both, transmitting and receiving antenna have vertical polarization. While the transmitting antenna is kept in a fixed position at a height of $1 \mathrm{~m}$, the receiving antenna is moved to 301 positions along a linear baseline with a distance to the transmit antenna varying from 0.25 to $6.25 \mathrm{~m}$ at the same height of $1 \mathrm{~m}$. In Figure 1 a picture of the investigated scenario including the baseline and the determined propagation paths for a receiver distance of $3.25 \mathrm{~m}$ is shown.

\section{Path Loss Exponent and Channel Capacity}

The first quantity of interest is the path loss, which is defined (in $\mathrm{dB}$ ) as

$$
P L=-10 \log _{10}\left(\frac{\bar{P}_{R}}{\bar{P}_{T}}\right)
$$

with $\bar{P}_{T}$ being the total transmitted and $\bar{P}_{R}$ being the total received power over the entire occupied frequency range. The basic log distance path loss model assumes that the logarithm of the received power decreases linearly with the logarithm of the distance and hence follows the law

$$
P L(d)=P L\left(d_{0}\right)+10 \eta \log _{10}\left(d / d_{0}\right)
$$

with $d_{0}$ being an arbitrary reference distance and $\eta$ being the path loss exponent. The second quantity of interest is the channel capacity, which is calculated here by sub-dividing the regarded frequency range into small bins, which can be interpreted as individual AWGN channels, provided that the bin size is smaller than the coherence bandwidth [3]. The total channel capacity results in

$$
C=\Delta f \sum_{i=1}^{L} \log _{2}\left(1+\operatorname{SNR}\left(f_{i}\right)\right)
$$

with $\Delta f$ being the bin size, which is chosen to $5 \mathrm{MHz}$ (the coherence bandwidth of the scenario is in the order of $10 \mathrm{MHz}$ ), $L$ being the total number of bins, which is equal to 1500 for the conducted simulations, and $S N R$ representing the signal to noise ratio, which is calculated for each bin individually assuming a transmit power spectral density according to the FCC indoor limit of $-41.3 \mathrm{dBm} / \mathrm{MHz}$ EIRP, no receiving antenna gain, and thermal noise with power spectral density $k \cdot T$ and a room temperature of $T=300 \mathrm{~K}$ disregarding the receiver noise figure.

\section{Simulation Results}

In Figure 2 the total received power in the investigated scenario dependent on the normalized receiver distance $d / d_{0}$ (both on logarithmic scales) is shown. 


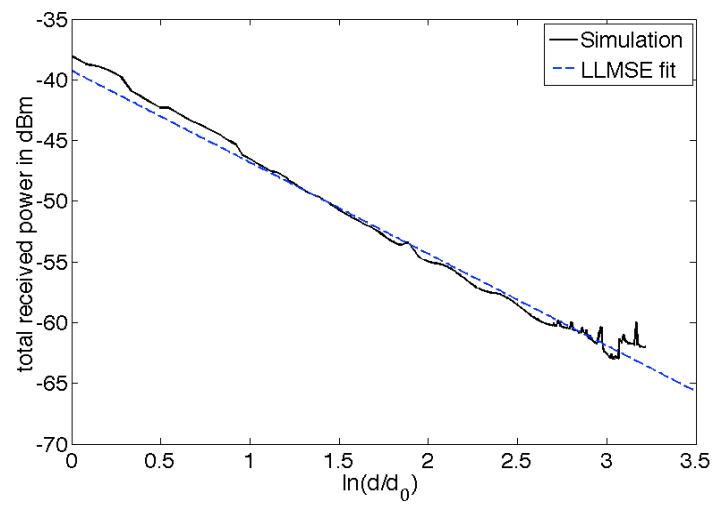

Figure 2: Total received power vs. normalized distance

The calculated LLMSE fit can be expressed similarly to (2) as

$$
\bar{P}_{R}(d)=\left(-39.2-10 \cdot 1.74 \cdot \log _{10}\left(d / d_{0}\right)\right) \mathrm{dBm}
$$

and hence the attenuation exactly shows the typical log distance path loss behavior with a path loss exponent of $\eta=1.74$ even though the investigated propagation scenario is based on many multi-path components which would cause deep fading in case of narrowband signals.

In Figure 3 the corresponding channel capacity is plotted dependent again on the normalized receiver distance.

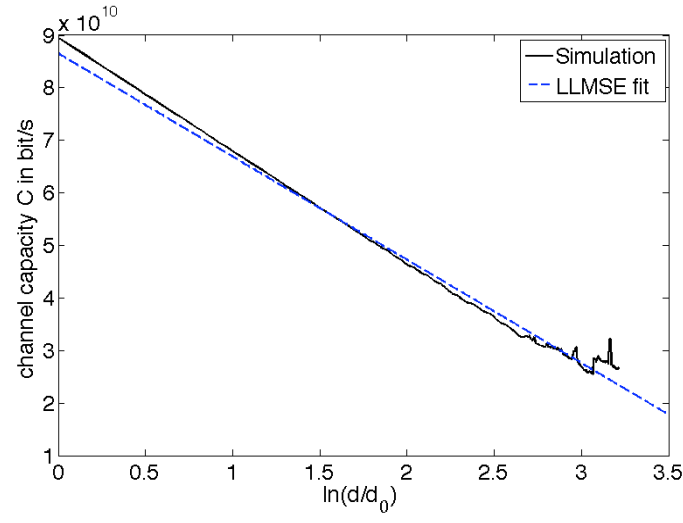

Figure 3: Channel capacity vs. normalized distance

It can be observed, that the (linear) channel capacity follows a similar linear behavior like the logarithm of the received power but with a different slope. The LLMSE fit results in:

$$
C(d)=\left(8.66-1.96 \cdot \ln \left(d / d_{0}\right)\right) \cdot 10^{10} \mathrm{bit} / \mathrm{s}
$$

In order to derive a relation between the path loss exponent and the capacity decrease, we use $\Delta f=B / L$ and $H(f, d)$ representing the channel transfer function at the frequency $f$ and distance $d$ : 


$$
C(d)=\frac{B}{\ln (2) \cdot L} \sum_{i=1}^{L} \ln \left(1+\frac{P_{T}\left|H\left(f_{i}, d\right)\right|^{2}}{P_{N}}\right)
$$

Applying Jensen's formula we obtain

$$
C(d) \leq \frac{B}{\ln (2)} \ln \left(1+\frac{1}{P_{N} \cdot L} \sum_{i=1}^{L} P_{T}\left|H\left(f_{i}, d\right)\right|^{2}\right)=\frac{B}{\ln (2)} \ln \left(1+\frac{\bar{P}_{R}(d)}{\bar{P}_{N}}\right)
$$

and assuming $\bar{P}_{R}(d) / \bar{P}_{N}>>1$ and normalizing to the reference distance $d_{0}$ gives

$$
C(d) \leq \frac{B}{\ln (2)}\left(\ln \left(\bar{P}_{R}\left(d_{0}\right)\right)-\ln \left(\bar{P}_{N}\right)\right)-\underbrace{\frac{\eta B}{\ln (2)}}_{=\alpha} \ln \left(d / d_{0}\right) .
$$

Hence we have found an empirical log distance law for the channel capacity with a slope $\alpha$ that is directly related to the path loss exponent $\eta$. Inserting $\eta=1.74$ obtained from (4) we get $\alpha=1.88 \cdot 10^{10} \mathrm{bit} / \mathrm{s}$ which corresponds very well to the result of the LLMSE fit in (5) with $\alpha=1.96 \cdot 10^{10} \mathrm{bit} / \mathrm{s}$.

\section{Conclusions}

Based on the results of the simulation of a deterministic multi-path propagation scenario it has been shown that for ultra wideband signals the received power does not show any fading characteristics but follows the simple log distance path loss model even in a dense multi-path environment. Furthermore, a similar log distance law has been derived for the channel capacity and a direct relation between the path loss exponent of the log distance path loss model and the capacity decrease has been found.

\section{Acknowledgement}

This work has been supported by the DFG (German Science Foundation) with the grants BO 1734/9-1 and WI 1044/22-1.

\section{References:}

[1] S. Sato and T. Kobayashi, "Path-loss Exponents of Ultra Wideband Signals in Line-of-Sight Environments", IEEE International Symposium on Spread Spectrum Techniques and Applications, pp. 488-492, Sydney, Australia, Aug.-Sept. 2004.

[2] C. Sturm, W. Sörgel, T. Kayser, W. Wiesbeck, "Deterministic UWB Wave Propagation Modeling for Localization Applications based on 3D Ray Tracing“, IEEE MTT-S International Microwave Symposium, pp. 2003-2006, San Francisco, California, June 2006.

[3] A. Goldsmith, Wireless Communications, Cambridge University Press, 2005. 